\title{
Situated Cognition \& Culture of Learning to Re-Conceptualize the Teaching-Learning Paradigm in the Field of Engineering and Technology in Bangladesh
}

\author{
Jahidul Arafat ${ }^{1}$, Mohammad Ahsan Habib ${ }^{2}$ and Che Kum Clement ${ }^{3}$ \\ ${ }^{I}$ Researcher, HT Research and Consultency, UK. \\ ${ }^{2}$ Department of Information and Communication Technology, Mawlana Bhashani Science and Technology \\ University, Bangladesh \\ ${ }^{3}$ Department of Technical and Vocational Education, Islamic University of Technology, OIC, Bangladesh
}

\begin{abstract}
Learning to teach is a complex process. On it, this paper discussed and examined on how a "learnreflect-judge" component of a teaching participation and reflection might achieve this. It presents a new unified teaching learning model named "Teaching learning Model to Reveal the Innovation and Sustainability". It integrates a prospective module named Self Generation Module and a new assessment approach named Spider wire Approach to aid the better management of T-L scenario in the field of engineering and technology in Bangladesh. Implication of this model at real time class room situation results with better management of learning and learning satisfaction.
\end{abstract}

Keywords: learn-reflect-judge loop, Self-Generation Module, Hypo Module, $360^{\circ}$ coverage, total effectiveness.

\section{Introduction}

The oath of empowering the robust manpower has caused the Bangladesh education system to become accountable for every individual's academic progress irrespective to their socioeconomic status, ethnicity, language or any other factors that may cause diversity in learning. Along with this system of accountability came an increased focus on the single most institutional influence in education, teachers: to improving the quality of teaching in order to increase the achievement levels of students. The efforts to improve the quality of education have been directed at addressing teaching methods and teacher motivation.

Most teachers develop their classroom skills fairly early in their teaching careers. Teachers entering the profession may find their initial teaching efforts stressful, but with experience they acquire a repertoire of teaching strategies that they draw on throughout their teaching. The particular configuration of strategies a teacher uses constitutes his or her "teaching style". While a teacher's style of teaching provides a means of coping with many of the routine demands of teaching, there is also a danger that it can hinder a teacher's professional growth. How can teachers move beyond the level of automatic or routines responses to classroom situations and achieve a higher level of awareness of how they teach, of the kinds of decisions they make as they teach, and of the value and consequences of particular instructional decisions? One way of doing this is through observing other's teaching style and trying to adopt the best of them and reflect it in his/her own style where observation and reflection are using for bringing change as referred by Bobis\& Aldridge [6]. This approach to teaching can be described as "Teaching observation and Reflection" which this paper aims to inquire to gain a greater insight into the type of experiences that will assist beginning as well as the in-teaching teachers to translate their hidden teaching efforts to their teaching practices and to present how an observation and reflective view (Teaching Practice) of teaching can assist the teachers to discover the needs and develop their individual teaching style. This paper examines teaching sessions those were participated and later presented by the researcher himself during his master's course work in an attempt to reflect, improve it and share what has been learnt.

The remainder of the paper is structured as follows. Section 2 deals with a framework which is proposed. The identification of areas to focus on is discussed in several subsections of section 2 and based on the framework a new model of teaching innovation and reliability is proposed in section 3. Section 4 presents a quantitative analysis on the adoptability of the proposed framework where section 5 concludes the paper with some general comments.

\section{The Proposed Framework for Teaching Innovation and Sustainability}

Several papers are now available which highlighted various techniques to develop and test a prototype model of teaching innovation and learning $[13,17-19]$ to overcome various limitations of actor (teacher), listener (student) and Stage (the classroom) to ensure better reliability and innovation in learning. The proposed framework in this study is based on these earlier work and some of the aspects, techniques and approaches are 
similar to the previous studies and on the basis of the study experiences where we got to know many new things about real and in-time teaching learning. Briefly the suggested framework, which is presented schematically in figure 1 , entails the following.

As an initial step, certain areas on which measurements will be taken need to be identified. These focus areas are necessary to ensure that areas, important to all teachers, form the basis of a measuring tool for innovation and sustainability. It will also help a teacher to focus aspects to be measured, when developing his teaching strategies. A value focused approach (VFA) is considered here that will take into account the concern areas of teachers i.e. (a) what will be the shape of an effective plan to maximize the learning among students, (b) which way will be best suited for managing the teaching learning and (c) how he should assess the students who are very diverse in their culture, nature and learning habits. The wings of this approach are presented in the next sections.

The whole process will be revolved around the dotted circle which is the heart for teaching innovation and reliability named "Learn-Reflect-Judge". Because whatever a teacher is doing in the step a, b and c of VFA $\mathrm{s} /$ he need to learn whether anything need to be changed and if so how and why to reflect those changes in his own teaching style to maximize the students learning and simultaneously will be judge by the respective teacher or student or special panel responsible for it to help the teacher to know how better he could organize the teaching learning scenario. And this process will revolve in a circular fashion to create a loop line to cause no end towards betterment because expect creator no own is perfect, everyone needs changes and only these positive and productive changes can ensure the sustainability and reliability.

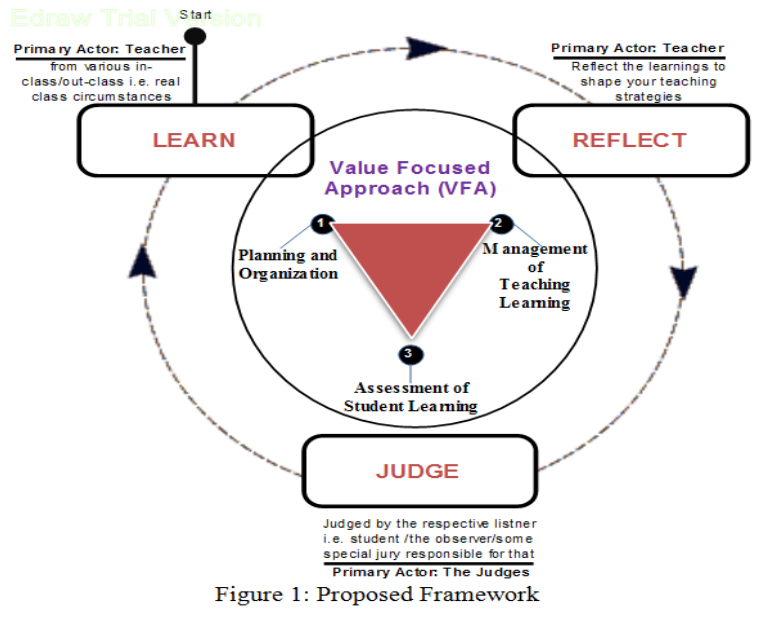

Figure 1: Proposed Framework

\subsection{Planning and Organization}

This is the real time planning place, where responsibilities belong to each particular teacher to gain the success in his teaching and to ensure the overall effectiveness. But does that teacher know what his best is? Or, what is the meaning of Best teaching? These may put them (the teacher) is a place of confusion, that's why to keep them away from it(the confusion) here this paper introduces a new scheme: "Self-Generated Module (SGM)" and belief that each of these teachers should go through this module to plan the best-to gain effectiveness in reflection(performance).

This self-Generated Module emphasizes on the following two basic steps which are details below namely (a) the hypothesis generation and (b) the spider wire approach to shape for effectiveness- which might be very helpful for the teachers to assist and guide them towards the effective classroom planning and Organization.

However, will we jump for planning without having a well-defined objective in our side? Of course not. This is the teacher who is playing the rule of coach for himself and thereby has to generate the objectives "what he is trying to do?" and "Will need tobe done?" to assist him in the phase of planning. And this objective can have the shape of the following forms:

\section{General Objective:}

- Discovering and developing the individual's (teachers) teaching style to achieve sustainability.

\section{Specific Objective:}

- Knowing what he (the teacher) want to accomplish is essential to help him with his teaching; 
- Virtually connecting what he or she wants to achieve with what may refracted back from the real time class room environment.

- Identifying what types of information he needs about his own teaching styles- because he is the self guided player.

These objectives will help us (as a teacher) to put our first step on the stage of planning to execute the Self generation module (SGM) from where we are trying to predict the future outcome by putting some controlled strategy oven them. Here the keyword prediction is directly related with the generation of hypothesis to foresee the future-is the $1^{\text {st }}$ stage of the SGM.

Stage 01: Hypothesis Generation- in this stage the teacher need to randomly choose any of the hypothesis that he seems to be helpful for the planning phase and this paper suggest that, teacher's can module these random selections into following HypoModules (HM): (a) Pattern of Behavior before class begin (b) Pattern of Behavior at the beginning of class (c) Pattern of classroom talking (d) Pattern of Classroom movement (e) Pattern of Eye Contact (f) Pattern of using the Black board and other audio visual equipment (g) Pattern of Questions and Directions (h) Pattern of Voice and Mannerism and (i) Pattern to End a class

These HM's will help a teacher to get the visualization on the whole process, where contents of these HM's depict the areas which need to be focused on when going for plan to ensure the high coverage rate on learning or effectiveness.

Now, after the planning has done, now teachers have to use the "Spider Wire Approach" as proposed in this paper to make interlinks among all of the enlisted HypoModule's for the purpose of effective organization of Teaching-Learning and for sustainability and reliability. The spider wire implementation of the HMs is shown in figure 2.

Stage 02: The Spider Wire Approach- This Spider Wire approach is a new innovation in the TeachingLearning scenario that is proposed in this paper and the paper strongly belief that this participation-\&-reflection strategy will help the teacher to gain effectiveness in the organization of the T-L when he will go for participation (perform) and when he will get the reflection back (classroom feedback)-to get an insight in "How to gain the success in teaching?", "How well need to be the T-L Plan?", and "Why the designed plan are not seeing the face of success?" and "How to update them (the T-L plan)?"

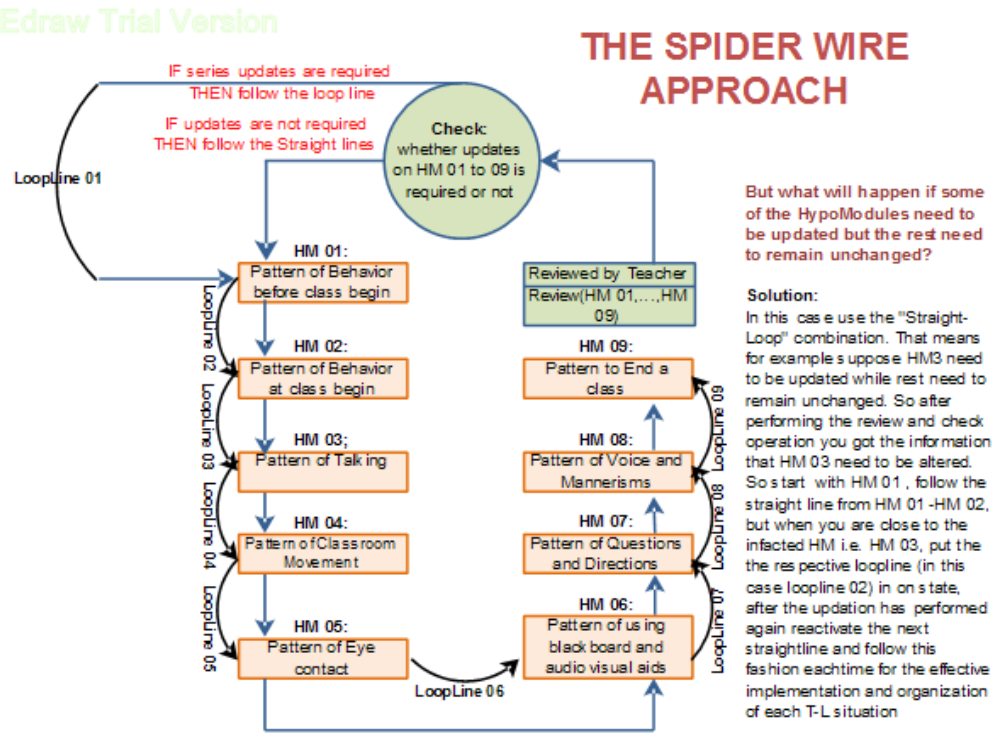

Figure 2: The Spider-Wire (SW) Approach for effective organization of T-L.

\subsection{Management of Teaching Learning}

Management of teaching learning means how a teacher is going to manage the environment of T-L to ensure the better deliverability of the day's concept. There are lot of ways and strategies to ensure the effectiveness in the concept delivery process, but we have to organize the teaching strategies in such a way that it will help to maximize the use of allocated instructional time to increase the student's learning. Here this paper proposed some of the mathematical strategies that may be helpful for those who are going to be the next 
generation teacher and those who are already in the teaching profession on the basis of the Spider wire approach that proposed above. And these are:

a. Management of Teachning Learning, MTL = Proper Integration Between (HM A, B, C, D, E, F, G, H, I) means proper management in each of the HM's that drawn above will ensure not only in time class room management but will also maximize the outcomes of learning by its innovation and reliability.

b. Executing (Spider Wire Seheme) $\rightarrow$ to readjust the proposed HM's (if necessary) to ensure the sustainable growth in the Learning and teaching process.

\subsection{Assessment of Student Learning}

The main motto of this section is to determine "How well did students learn and achieve their learning objectives?" .This determination can be done on the basis of the following three domains, where wing of the judgment is spread over to gain information about the student's level of attainment.

- Domain 01: Learner Participation

- Domain 02: Learning and Attainment

- Domain 03: Learner Differentiation, Equality and Diversity

Domain 01: Learner participation- which can be further evaluated by having in-depth views on the following aspects [1, 3, 12-14]: Student's (a) Attendance and Punctuality (b) Attentiveness and Engagement in learning (c) Motivation towards learning (d) Peer learning and Independent learning.

Domain 02: Learning and Attainment- The learning and attainment rate can be estimated by having an indepth view on the following aspects [1, 2, 14]: (a) Assign purposeful on-task activity to student (b) Assess student's progress in Learning.

Domain 03: Learner differentiation, Equality and Diversity- "Learners are of diverse nature"-this truth reveals that when the teacher are intended to judge them (student), a single judgmental tool may not be an effective one to cover all. That's why; to estimate the learner differences and inequality as a teacher one need to have an in-depth view on the followings [1,2,12]: (a) Differentiated and appropriate tasks (b) Differentiated monitoring and assessment Technique (c) Variety of teaching \& learning strategies.

However, these also ties into different approaches to students. To do so, this paper belief to follow the following care-modules $(\mathrm{CM})$ as proposed in several journals $[1,3,6-10,13-16]$ to ensure the maximum caring for individual's learning.

CM A Maintains Student Interest to the End of the session

CM B Makes clear what is expected from the class

CM C Encourages student to think critically during the class

CM D Encourages the student to relate what $\mathrm{s} / \mathrm{he}$ has heard/seen to their own experience and/or specific problems in the field

CM E Encourages the students to offer their own knowledge and/or opinions

CM F Leaves the student feeling stimulated to think and learn more about the subject

And all these will ultimately be helpful for the respective teacher to find out which HM is lacking behind and how the SW approach should be executed while leaving two hypothetical question for them (teacher): (a) do you aim above their heads and encourage them to stretch up to reach this higher bar? Or (b) do you get down below them, as it were, and push them up from behind? As a student of the Teaching Method I found that teaching and teacher's success relies with the second one; but why on it? Because you are the teacher of your students where each having their own traits and pattern of understanding. Get down to their level will help you to gain an insight of each of them to treat them accordingly-thereby to maximize their learning.

And to do so this paper presents a cross functional layered chart named "Functional Layered Chart of Assessment" (see Figure 3) which is shown below to depict the relationship among these three domains and to assist the teachers to know how, when and where to make the utmost use of the assessment tools to ensure the maximum caring for individual's learning.

This cross functional flow chart is divided into 3 domains and 7 areas, where the domains are: (a) Learner Participation (b) Learning and Attainment and (c) Learner Differentiation, equality and Diversity. And the areas are: 
- Area 01: Is a lone function of "Domain 01: Participation", reflect how as a teacher you can successfully assess the student's participation to reflect their learning.

$\bullet$

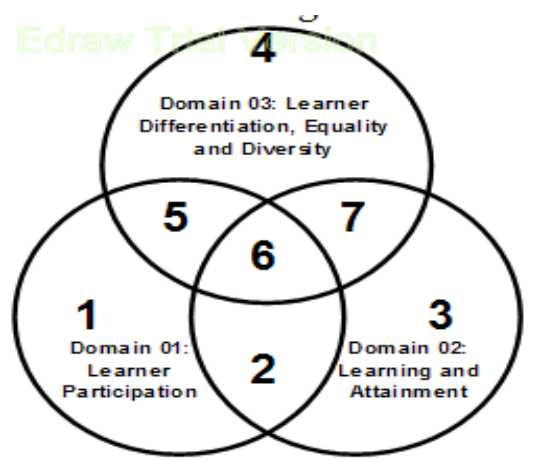

Figure 3: Functional layered Chart of Assessment

- Area 02: Is a composite function of Domain 01 and 02. Here these two domains overlap with each other to reflect the impact of the Learner's participation in their learning and attainment. And this is the truth that we found in our observations that the class which one was more participatory perform much better than the class which was a less participatory one in assessment (which was taken by their teachers).

- Area 03: Is a lone function of Learning and Attainment, reflects how the student's learning and attainments are assessed by the respective teachers i.e. which measurement tools they used to do it.

- Area 04: Is a lone function of Domain 03, reflects which strategies teacher's need to adopt to assess the different and diverse natures of students in a very effective way, to extract the best out of them.

- Area 05: Is a composite function of Domain 01 and 03, to reflect the impact of learner differences and diversity in their degree of participation. And this is the truth that I found in my observations that the classes where the respective teachers used various strategies to assess the diverse nature of students were more successful to get the most out of them than the class where teacher used a single utmost strategy to judge all.

- Area 06: Is a composite function of Domain 01, 02, and 03, where these three domains overlap with each other. This is our target area which reflects how learner's learning and attainments are influenced by their participation and how the degree of participation is affected by the diverse nature of the learners.

- Area 07: Is a composite function of Domain 02 and 03, where these overlaps with each other to reflect the impact of students nature on their pattern of learning and attainment, and to make the teacher's aware on what tools they need to use to gain the utmost from each student/learner.

If a teacher gone through these 3 domains and 7 areas to make a proper care and assessment of learning according to the demand of the situation, s/he will gain success in caring for individual learning-to maximize it to gain the teaching reliability and overall sustainability. As a concluding remark of this section, we want to quote that, "Learning and Attainment depends on the Learner's participation, where Learner's differences, equality and diversity have some direct influence on the degree of their participation in learning and as a teacher we need to focus on it to gain the utmost from our students to ensure the best use of the adopted assessment tools."

\section{The Proposed Teaching Learning Model to Reveal the Innovation and Sustainability}

As we have to go through 3 different engineering institutions of Bangladesh to attend 100 classes of 60 different teachers where every institutions have their own culture and values. There students from various sections of diverse natures and traits merging together for the purpose of learning. So as a trainee teacher we got a great chance to gain ideas on what we need to do and don't need to do while facing the real time class room situations. Hence a bundle of Teaching innovation models or strategies are already is in place but due to their scattered alignment and poor coordination lacking behind to attain the $360^{\circ}$ coverage in the learning and teaching. Keeping all these in consideration, this paper present a new model for innovation and sustainability named "Teaching learning Model to Reveal the Innovation and Sustainability" based on the framework as discussed above (see Figure 04). However, this proposed TLMIS model begins with assessing the student's characteristics in respect to their pace, class and prior achievement which will help the teacher to set down their expectation regarding the respective student's level of attainment and once after it has been done teacher can execute the above discussed Spider Wire Approach (SWA)in the following three phases (a) Assessment of student's learning (b) Management of Student's Learning and (c) Planning and Organization in the form of 
his/her teaching practicum while incorporating the above discussed self-Generation Module (SGM). And this thereby will assist them to be best in their preparation and to pull out the best from the student's learning. Furthermore in the form of feedback, execution of this SWA in these three phases will also assist the teacher in remodeling his/her expectation on that student and treat them accordingly to assist in maximizing their learning outcomes.

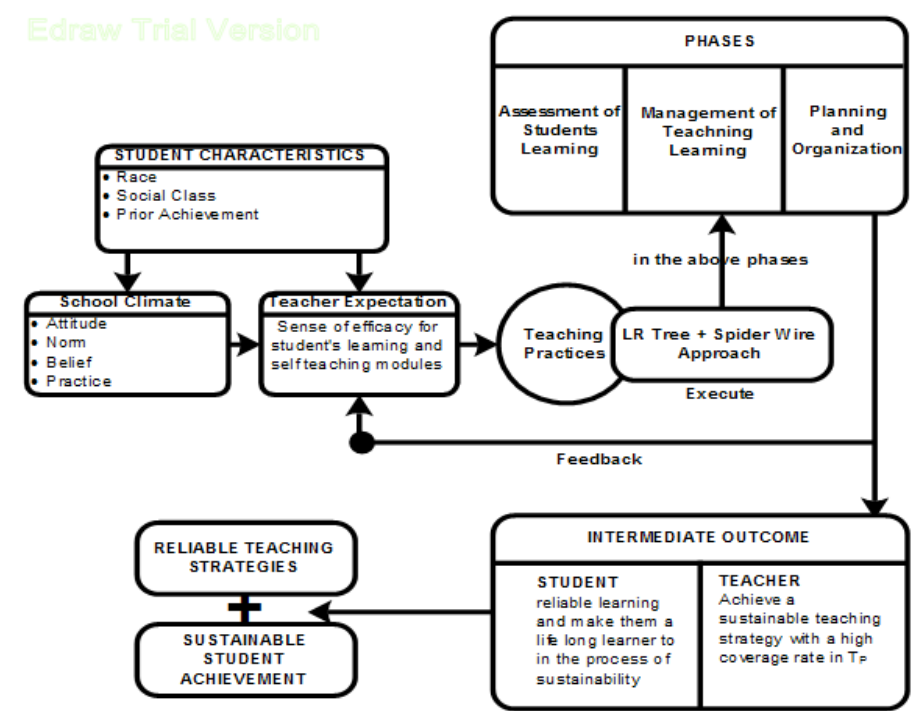

Figure 04: Teaching learning Model to Reveal the Innovation and Sustainability (TLMIS)

However, the immediate outcome of the implementation of this proposed model is that it will assist student in attaining reliable learning and will transform them a lifelong learner to contribute in the process of sustainability while assisting teacher indeveloping a sustainable teaching strategy with a high coverage rate in Task of "Practice Teaching", $\mathrm{T}_{P}=$ \{Extend of preparation, skill of presentation, skill of developing the concept and communication with the students, use of learning resources and its impact, class management and rapport with the students, feedback and assessment of student learning\} and thereby to contribute to this progressive studentlearning process. Based on this theme a teacher can segment his/her dos and don'ts into four possible phases as further suggested byseveral researchers [9,12,15](a) Planning and Preparation (b) The Classroom Environment (c) Instruction (e) Professional Responsibilities, which this study believe will further revamp the proposed process in contribution to the T-L outcomes and stability.

\section{Quantitative Survey Result}

In analyzing the teaching learning reliability and sustainability performance of the TLMIS approach, the responses of 6 senior institution administrators- the majority of whom were principle or head of departments along with 100 academic personnel at 3engineering institutions of Bangladesh were synthesized, where the selection of samples are done on convenient sampling methodology considering the time and budget constraints of the research. The existing teaching learning trends of these institutions were physically observed in a qualitative manner on inductive thought by the respective researcher and in the light of the findings the TLMIS approach is developed and later the surveyed institutions were asked to implement this newly developed teaching innovation scheme in their arena. The impact of the implementation status of this TLMIS approach at those institutions were further analyzed against four major survey questions to assess respondents' opinions on the success of their T-L outcomes Likert scale ranging from: $1=$ strongly agree, $2=$ agree, $3=$ Disagree, and $4=$ Strongly Disagree) along with two dedicated questionnaire sets for both the senior administrators and for the academic personnel with details feature on acceptability, concerns and development which however has been blended on the following four major concerns of the study as depicted below:

a) How would you characterize your education program's success?

b) Are teachers more successful in their teaching than before?

c) Are the students learning outcomes much higher than before?

d) Is the overall teaching learning environment more reliable than 1 year ago? 
Table 1: Impact of TLMIS's Implementation Status over Institution's T-L Outcomes

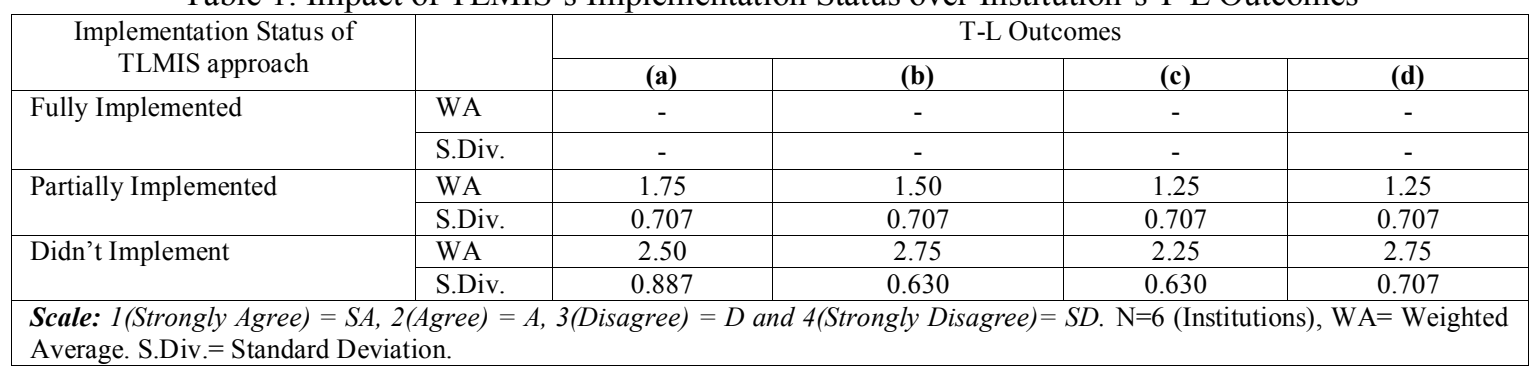

Table 1 shows that because of the shortage of time and resource availability none of the institutions could implement this proposed solution fully but institutions which has implemented the proposed TLMIS approach in their arena at least partially rates their T-L outcomes higher than those which didn't. However, they also rated high on their teaching learning environment's reliability that it was about 1year ago and also reported high on their teacher's success and learner's outcomes. These thereby dictate the significance of having this newly developed Teaching Learning management model in the campus arena to gain robustness in the process of learning and teaching.

\section{Conclusion}

A major concern for teaching reliability and sustainability has been the need to find more effective ways to address the apparent observation/practice gap and better prepare our teachers to master the realities of teaching. Although such procedural matters are important, beginning teachers' preoccupations with them generally means that they are unable to consider new, more cognitively demanding, teaching approaches advocated in key policy documents during their teacher preparation programs. A challenge facing teacher educators is to design teaching-learning environments that will empower beginning teachers to translate theory into their practice more effectively. A solution to these challenges is the "Teaching learning Model to Reveal the Innovation and Sustainability" as presented in this paper to help a teacher to become a self guided player with its enriched features of Self Generation Module (SGM). This SGM composed 9 HypoModules (HM) to help the teachers to make effective plans to execute his teaching arms and to thereby foster the learning and a Spider wire tool to assist them digging out where he is lacking behind to fill the gaps up and to thereby ensure a robust management and assessment of T-L situation and scenario. And these proposed model is not just a mere theory based on fantasy rather was presented to the surveyed institutions to execute it in their arena to facilitate their teachers with these enriched features to enjoy the flavor of teaching and to let the student enjoy their learning environment and the quantitative outcomes were positive.

In this proposed T-L approach, the degree used for merging the Teaching and learning concerns with that of the students nature and teacher's belief and constraints are derived dynamically based on needs of T-L innovation and reliability for the $21^{\text {st }}$ century's knowledge hoarder. For these reasons, this T-L solution is able to present the institution's education concerns from a holistic position. The quantitative survey results confirms that the institutions which had adopted this proposed teaching learning solution to facilitate their faculty members in maximizing their teaching outcomes and thereby to incorporate the learning dimensions feeling their overall teaching learning environment more reliable than six months ago before adopting this. They also rated their teacher's teaching and education program's success as well as the students learning outcomes much higher than that of others. This thereby increases the application area of the teaching learning approaches where the robustness and dynamisms are needed for innovation and sustainability.

\section{References}

[1]. Aldridge, S., \&Bobis, J. (2001). Multiple learning contexts: A vehicle for changing preservice teachers' mathematical beliefs, knowledge and practices. In J. Bobis, B. Perry \& M. Mitchelmore (Eds.), Numeracy and Beyond, (Proceedings of the Twenty-Fourth Annual Conference of the Mathematics Education Research Group of Australasia, Sydney, pp. 43 -49), Sydney: MERGA.

[2]. Australian Association of Mathematics Teachers. (2002). Standards for excellence in teaching mathematics in Australian schools, Adelaide: AAMT.

[3]. Australian Education Council. (1990). A national statement on mathematics for Australian schools. Canberra: Curriculum Corporation.

[4]. Black, P., \&Wiliam, D. (1998). Inside the black box: Raising standards through classroom assessment. London: King's College London School of Education.

[5]. Board of Studies, New South Wales. (2002). Mathematics K-6, Sydney: Board of Studies.

[6]. Bobis, J., \& Aldridge, S. (2002). Authentic learning contexts as an interface for theory \& practice. In A. Cockburn \& E. Nardi (Eds.), Proceedings of the 26th Conference of the International group for the Psychology of Mathematics Education (pp. 121-127).PME: University of East Anglia, Norwich.

[7]. Brown, J., Collins, A., \&Duguid, P. (1989). Situated cognition and the culture of learning. Educational Researcher, 18(1), 32-42. 
[8]. Korthagen, F., \&Kessels, J. (1999). Linking theory and practice: Changing the pedagogy of teacher education. Educational Researcher, 28(4), 4-17.

[9]. Liston, D., Whitcomb, J., \&Borko, H. (2006). Too little or too much: Teacher preparation and the first years of teaching. Journal of Teacher Education, 57 (4), 351-358.

[10]. Moore, R. (2003). Reexamining the field experiences of preservice teachers, Journal of Teacher Education,54(1), 31-42.

[11]. National Council for Teachers of Mathematics. (2000). Principles and standards for school mathematics. Reston, VA: NCTM.

[12]. New South Wales Department of Education and Training. (2003). Quality teaching: Discussion paper, Sydney: NSWDET.

[13]. New South Wales Institute of Teachers. (2006). Professional teaching standards. Sydney: NSW Institute.

[14]. Putnam, R., \&Borko, H. (2000). What do new views of knowledge and thinking have to say about research on teacher learning? Educational Researcher, 29(1). 4-15.

[15]. Shavelson, R. (2006). On the integration of formative assessment in teaching and learning: Implications for new pathways in teacher education. In F. Oser, F. Achtenhagen, \& U. Renold (Eds.), CompetenceOriented Teacher Training (pp. 63-78). Rotterdam: Sense Publishers.

[16]. Tobin, K., \& Roth, W. (2006). Teaching to learn: A view from the field. Rotterdam: Sense Publishers.

[17]. Wilson, S., Floden, R., \&Ferrini-Mundy, J. (2002). Teacher preparation research: An insider's view from the outside. Journal of Teacher Education, 53(3), 190-204.

[18]. Zeichner, K., \&Tabachnick, B. (1981). Are the effects of university teacher education washed out by school experiences? Journal of Teacher Education, 32, 7-11. 